\title{
Curcumin induces therapeutic angiogenesis in a diabetic mouse hindlimb ischemia model via modulating the function of endothelial progenitor cells
}

Jinzhi You ${ }^{1+}$, Jiacheng Sun ${ }^{1+}$, Teng Ma ${ }^{1+}$, Ziying Yang ${ }^{1+}$, Xu Wang ${ }^{1}$, Zhiwei Zhang ${ }^{1}$, Jingjing Li ${ }^{1}$, Longgang Wang ${ }^{1}$, Masaaki $\mathrm{il}^{2}$, Junjie Yang ${ }^{1 *}$ and Zhenya Shen ${ }^{1 *}$

\begin{abstract}
Background: Neovascularization is impaired in diabetes mellitus, which leads to the development of peripheral arterial disease and is mainly attributed to the dysfunction of endothelial progenitor cells (EPCs). Previous studies proved the promotional effect of curcumin on neovascularization in wound healing of diabetes. Thus, we hypothesize that curcumin could promote neovascularization at sites of hindlimb ischemia in diabetes and might take effect via modulating the function of EPCS.

Methods: Streptozotocin-induced type 1 diabetic mice and nondiabetic mice both received unilateral hindlimb ischemic surgery. Curcumin was then administrated to the mice by lavage for 14 days consecutively. Laser Doppler perfusion imaging was conducted to demonstrate the blood flow reperfusion. Capillary density was measured in the ischemic gastrocnemius muscle. In addition, angiogenesis, migration, proliferation abilities, and senescence were determined in EPCs isolated from diabetic and nondiabetic mice. Quantitative PCR was then used to determine the mRNA expression of vascular endothelial growth factor (VEGF) and angiopoetin-1 (Ang-1) in EPCs.

Results: Curcumin application to type 1 diabetic mice significantly improved blood reperfusion and increased the capillary density in ischemic hindlimbs. The in-vitro study also revealed that the angiogenesis, migration, and proliferation abilities of EPCs and the number of senescent EPCs were reversed by curcumin application. Quantitative PCR confirmed the overexpression of VEGF-A and Ang-1 in EPCs after curcumin treatment.
\end{abstract}

Conclusion: Curcumin could enhance neovascularization via promoting the function of EPCs in a diabetic mouse hindlimb ischemia model.

Keywords: Curcumin, Diabetes mellitus, Endothelial progenitor cells, Hindlimb ischemia, Neovascularization

\section{Background}

Diabetic mellitus has a high risk of developing peripheral arterial disease (PAD) and critical limb ischemia [1], which is mainly attributed to hyperglycemia-induced impaired neovascularization. Neovascularization, including angiogenesis and vasculogenesis, plays a crucial role in the delivery of oxygen, nutrients, and other mediators at

\footnotetext{
* Correspondence: jjyang@suda.edu.cn; uuzyshen@aliyun.com ${ }^{\dagger}$ Equal contributors

${ }^{1}$ Department of Cardiovascular Surgery of the First Affiliated Hospital \& Institute for Cardiovascular Science, Soochow University, Suzhou, China Full list of author information is available at the end of the article
}

the injury and ischemic sites. Angiogenesis is a progress of stimulation, promotion, and stabilization of new blood vessels that involves major angiogenic factors such as vascular endothelial growth factor (VEGF) and angiopoetin-1 (Ang-1), but their levels decrease in diabetes [2, 3]. Vasculogenesis involves participation of endothelial progenitor cells (EPCs), a cell type that is capable of differentiating into functional endothelial cells and replacing the injured endothelial cells $[4,5]$. During vasculogenesis, EPCs mobilize from bone marrow (BM), home to the ischemic site, differentiate into endothelial cells, and participate in endothelial repair $[6,7]$. 
However, the number and function of EPCs are reduced in diabetes-associated peripheral vascular disease [8], which is a major cause of impaired vascular regeneration/maintenance under hyperglycemia conditions [9].

There are limited medical therapies to improve angiogenesis in diabetes associated with PAD [10]. Recently, Kant et al. [11] indicated that curcumin had a proangiogenic effect on wound healing in type 1 diabetes. Curcumin $\left(\mathrm{C}_{2} \mathrm{H}_{20} \mathrm{O}_{6}\right)$ or diferuloylmethane is a bright-yellow chemical isolated from the roots of Curcuma Longa (turmeric), and has been widely used and studied over the past 60 years in the treatment of numerous diseases [12]. In addition to the proangiogenic effect, evidence also showed that curcumin had antiangiogenic effects on pituitary adenomas and hepatic cancer [13, 14]. Therefore, curcumin exhibited contradictory results in different settings of diseases.

Considering the limited studies of curcumin in diabetic ischemia, the present study was conducted to investigate the potential of curcumin on neovascularization in diabetic ischemia as well as the possible mechanism.

\section{Methods}

\section{Animals}

Healthy male C57/BL6 mice were housed in standard polycarbonate cages in the Animal Facility of Soochow University. Animals were maintained on a 12-hour light/ dark cycle as well as provided with free access to feed and water. All animals received humane care in accordance with the Guidelines for the Care and Use of Research Animals established by Soochow University.

\section{Type 1 diabetes and hindlimb ischemia induction}

Type 1 diabetes was induced by intraperitoneal injections of streptozotocin (Sangon Biotech, Shanghai, China). Male C57/B6 mice (4-6 weeks of age) received an injection of citrate buffer $(4.92 \mathrm{~mol} / \mathrm{ml}$ sodium citrate, $\mathrm{pH} 4.2-4.5)$ or streptozotocin $(50 \mathrm{mg} / \mathrm{kg})$ dissolved in sterile citrate buffer for 5 days consecutively. The blood glucose level was measured 7, 14, and 21 days after the injection. Mice with a blood glucose level $>$ $12.0 \mathrm{mmol} / \mathrm{L}$ were considered diabetic and selected for experiments.

Three weeks after diabetes induction, all nondiabetic and diabetic mice received unilateral hindlimb artery devascularization. Mice aged 6-8 weeks were anesthetized with $160 \mathrm{mg} / \mathrm{kg}$ pentobarbital by intraperitoneal injection. During the operation, the superficial and deep femoral vessel, the common femoral vessel and its abdominal branches were ligated and excised to generate hindlimb ischemia as described previously [15]. The right femoral artery was exposed but not dissected to serve as the nonischemic control.

\section{Groups and curcumin treatment}

Animals were divided equally into four groups. Twenty mice were used in this study, namely five mice for each group. In the nondiabetes group, mice with euglycemia were treated with $300 \mu \mathrm{l}$ of sterile saline by lavage once a day for 14 days. In the diabetes with saline treatment group, sterile saline $(300 \mu \mathrm{l})$ was applied to diabetic mice by lavage once a day for 14 days. In the diabetes with olive oil treatment group, totally $300 \mu \mathrm{l}$ of extra virgin olive oil was administrated by lavage to each mouse once a day for 14 consecutive days. In the diabetes with curcumin treatment group, $1000 \mathrm{mg} / \mathrm{kg}$ curcumin (SigmaAldrich, St. Louis, MO, USA) in $300 \mu$ l of olive oil was applied to mice once a day for 14 days.

\section{Laser Doppler perfusion imaging}

Laser Doppler perfusion imaging (LDPI) (Perimed Instruments AB, Stockholm, Sweden) was conducted on mice immediately, 7 days, and 14 days after ischemic surgery. LDPI was used to measure the blood flow recovery ratio in ischemic hindlimbs:

$$
\text { Blood flow recovery ratio }=\frac{\text { Ischemic limb perfusion }(\text { left hindlimb })}{\text { Nonischemiclimb perfusion }(\text { right hindlimb })} \times 100 \%
$$

Colored histogram pixels indicated the blood reperfusion of ischemic and nonischemic limbs.

\section{In-vivo capillary density measurement}

The capillary density indicates the angiogenesis in the ischemic hindlimb. Generally, mice were sacrificed 14 days after hindlimb ischemic surgery to collect ischemic muscle samples, namely the left gastrocnemius muscle. Then muscle samples were embedded in OCT compound (Sakura, Torrance, CA, USA), frozen by liquid nitrogen, and cut by Leica CM 1950 Cryomicrotome (Carl Zeiss AG, Jena, Germany) at a thickness of $6 \mu \mathrm{m}$. Frozen sections were first washed in phosphate-buffered saline (PBS), and then stained with diluted fluorescein-IB4 (Invitrogen, Carlsbad, CA, USA) at room temperature for 1 hour. Samples were then washed in PBS again and sealed with a coverslip. A fluorescent microscope (Olympus, Tokyo, Japan) was used to examine the images.

\section{EPC isolation and characterization}

After ischemic muscle samples were collected, the bones were then separated for EPC isolation. The purified EPCs would be used directly for the following functional determination with no other treatment. The protocol of EPC isolation and culture was described previously [16, 17]. After the muscle sample was collected, the bones were then separated and smashed to collect bone marrow mononuclear cells (BM-MNCs). The bone sample includes hipbones, femurs, and tibiae, as well as 
shoulder bones, ulnas, vertebra, and sternum. EPCs were further isolated by layering the BM-MNCs on a density gradient (Histopaque 1083; Sigma) followed with centrifugation and were cultured in Endothelial Cell Basal Medium-2 (EBM-2; Lonza, Basel, Switzerland), supplemented with EGM-2 MV SingleQuots (Lonza). After 2 days of culture in a $37{ }^{\circ} \mathrm{C}, 5 \% \mathrm{CO}_{2}$ incubator, nonadherent cells were washed off by PBS while adherent cells were further incubated in fresh EBM-2 for 1 week before experiments.

Culture medium was gently washed off by PBS, and then cells were cultured in $5 \mu \mathrm{g} / \mathrm{ml}$ of DiI-Ac-LDL solution (Biomedical Technologies Inc., Stoughton, MA, USA) in EBM-2 for 4 hours at $37{ }^{\circ} \mathrm{C}$, with the dish wrapped in aluminum foil. The cells were then fixed by $4 \%$ paraformaldehyde (PFA)/PBS for $20 \mathrm{~min}$ at $37{ }^{\circ} \mathrm{C}$. After this, the cells were washed by PBS and DAPI staining solution (Beyotime Biotechnology, Shanghai, China) was added to stain the nuclei. After $10 \mathrm{~min}$ of incubation, staining solution were washed off by PBS and the sample was ready to be observed by fluorescent microscope.

\section{EPC tube formation assay}

The tube formation assay was carried out as described previously to determine the in-vitro tube incorporation potential of EPCs $[18,19]$. Briefly, human umbilical cord-derived endothelial cells (HUVECs; $1 \times 10^{4}$ cells/ well) and DiI-labeled (Invitrogen, Eugene, OR, USA) EPCs $\left(1 \times 10^{3}\right.$ cells/well) were first resuspended using EBM-2 $(100 \mu \mathrm{l})$, then seeded together on a 96 -well plate, with $50 \mu \mathrm{l}$ Matrigel matrix (BD, Bedford, MA, USA) added to each well beforehand, and incubated for $30 \mathrm{~min}$ at $37^{\circ} \mathrm{C}$. A fluorescent microscope was used to take morphology images, to count the number of DiIlabeled cells, and to measure the tube length.

\section{EPC transwell assay}

A modified Boyden chamber assay was used to examine the EPC migration activity [20]: $500 \mu \mathrm{l}$ of 15\% FBS/Dulbecco's modified Eagle's medium (DMEM; Gibco, New York, NY, USA) was added into the lower chamber. EPCs $\left(1 \times 10^{4}\right.$ cells/well $)$ in $200 \mu$ l serum-free medium were placed in the upper chamber, with $0.05-0.2 \%$ bovine serum albumin (BSA; Amresco, Englewood, CO, USA) in order to maintain the osmotic pressure. The chamber was incubated for 4 hours under $5 \% \mathrm{CO}_{2}$ at $37{ }^{\circ} \mathrm{C}$. Cells on the lower side of the chamber were fixed with PFA and stained with DAPI. The number of migrated cells was counted using a fluorescent microscope.

\section{EPC senescence detection}

EPC senescence was evaluated based on the quantity of senescence-associated $\beta$-galactosidase (SA- $\beta$-gal)-positive cells. SA- $\beta$-gal was stained using the senescence $\beta$ - galactosidase staining kit (Beyotime Biotechnology, Shanghai, China), according to the manufacturer's instructions [21]. The number of SA- $\beta$-gal-positive cells was determined by counting blue cells from at least 1000 cells per field.

\section{Colony-formation assay}

Methylcellulose-based medium (MethoCultM3236; Stem Cell Technologies, Vancouver, BC, Canada) was prepared with supplements as follows: VEGF (50 ng/ml; PeproTech, Rocky Hill, NJ, USA), SCF (100 ng/ml; PeproTech), IL-3 (20 ng/ml; PeproTech), EGF (50 ng/ $\mathrm{ml}$; PeproTech), bFGF (50 ng/ml; PeproTech), IGF-1 (50 ng/ml; PeproTech), and 30\% FBS. EPCs were cultured in this medium for 14 days under $5 \% \mathrm{CO}_{2}$ at $37^{\circ}$ C. A phase-contrast microscope (Olympus) was used for manual quantitative counting.

\section{Real-time reverse-transcriptase PCR analysis}

Real-time reverse-transcription polymerase chain reaction (RT-PCR) was used to determine the mRNA expression of VEGF and Ang-1 in EPCs and hindlimb muscle. Total RNA was extracted using Trizol reagent (Ambion by Life Technologies, Carlsbad, CA, USA) and then purified according to the manufacturer's protocol (QIAGEN, Valencia, CA, USA). cDNA was converted from extracted total RNA using the PrimeScript RT reagent kit (TAKARA, Tokyo, Japan). Then, $2 \mu \mathrm{l}$ of cDNA sample was used as a template for quantitative real-time PCR (RT-qPCR). qPCR was performed using Power Syber Green (Applied Biosystems, Foster City, CA, USA) and the StepOne-Plus real-time PCR system (Applied Biosystems) according to the manufacturer's guidelines. The endogenous housekeeping gene GAPDH was used to normalize the results. The sequences of primers used in $\mathrm{qPCR}$ are presented in Table 1 . The $2^{-\Delta \Delta C T}$ method was used to evaluate the relative quantification of changes in the expression of target genes [22].

\section{Statistical analyses}

All results are expressed as mean $\pm \mathrm{SD}$. The data were analyzed using GraphPad Prism 6.0 software, via Student's unpaired $t$ test for the difference between two groups or one-way ANOVA with Bonferroni correction for multiple group comparison. Differences were considered significant at $P<0.05$.

\section{Results}

Promoted in-vivo blood flow recovery in curcumintreated type 1 diabetic mice

A diabetic mouse ischemia model was constructed to examine the neovascularization ability with curcumin treatment. Blood perfusion was measured by LDPI at day 0,7 , and 14 after the ischemic operation, and the 
Table 1 Primer sequences for quantitative real-time PCR

\begin{tabular}{lll}
\hline Gene name & Forward primer $\left(5^{\prime}-3^{\prime}\right)$ & Reverse primer $\left(5^{\prime}-3^{\prime}\right)$ \\
\hline mVEGF-A & AGCACAGCAG & AATGCTTCT \\
& ATGTGAATGC & CCGCTCTGAA \\
mANG-1 & ATCTTGATAAC & TGTCGGCACA \\
& CGCAGCCAC & TACCTCTTGT \\
GAPDH & TGTGTCCGTC & ACCACCTTCTTA \\
& GTGGATCTGA & TGTCATCATACTT \\
\hline
\end{tabular}

recovery index was presented by the blood flow recovery ratio as mentioned previously [23] (Fig. 1a). In general, blood flow recovery was significantly delayed in the salinetreated and olive oil-treated groups, whereas the blood flow reperfusion in curcumin-treated group presented a dramatic elevation, exhibiting a similar recovery ratio to the nondiabetic group. Specifically, the blood flow recovery ratio in the olive oil-treated group was $16.93 \pm 3.60 \%$ at day 7 and $15.36 \pm 7.04 \%$ at day 14 (Fig. 1b, c). In comparison, the ratio in the curcumin group (day $7,37.17 \pm$ 9.83\%; day $14,56.99 \pm 13.02 \%$ ) had significantly increased $(P<0.05)$, indicating that curcumin could promote blood flow recovery in diabetic hindlimb ischemia.

Enhanced neovessel density of diabetic ischemic hindlimb In addition to LDPI, capillary density was further examined by immunohistochemistry at day 14 postoperatively. The immunofluorescent agent IB4 was applied to endothelial cells. IB4-labeled capillaries were manually counted in high- power fields (HPFs). Overall, EPCs isolated from diabetic mice with saline treatment and olive oil treatment both exhibited insufficient growth of neovessels (Fig. 2; saline group, $53.40 \pm 8.79 / \mathrm{HPF}$; olive oil group, $62.00 \pm 7.58 / \mathrm{HPF}$ ). On the contrary, enhanced neovascularization could be observed in the curcumin-treated diabetes group (111.40 $\pm 15.27 / \mathrm{HPF})$. These data demonstrate that the angiogenesis in diabetic ischemic hindlimb was augmented by curcumin treatment.

\section{Characterization of EPCs}

Cells were cultured in medium of $10 \%$ FBS/EBM-2MV for 7 days after isolation from mice BM. At day 3, adherent cells changed their morphology into a spindle shape. At day 7, most cells had changed their appearance and formed colonies as reported previously [5, 24] (Fig. 3a). Further investigation was conducted to determine the EPC characteristics by DiI-Ac-LDL uptake assay. Cells positive for uptake DiI-Ac-LDL observed by fluorescent microscope were considered EPCs (Fig. 3b).

\section{Augmented in-vitro tube incorporation activity of curcumin-treated EPCs isolated from diabetic mice} The tube formation assay was introduced to study the angiogenesis potential of EPCs according to former reports $[19,25]$. EPCs are not capable of forming the tube-like structure independently, but could integrate into the tube-like structure formed by HUVECs. The number of incorporated EPCs was counted manually. As

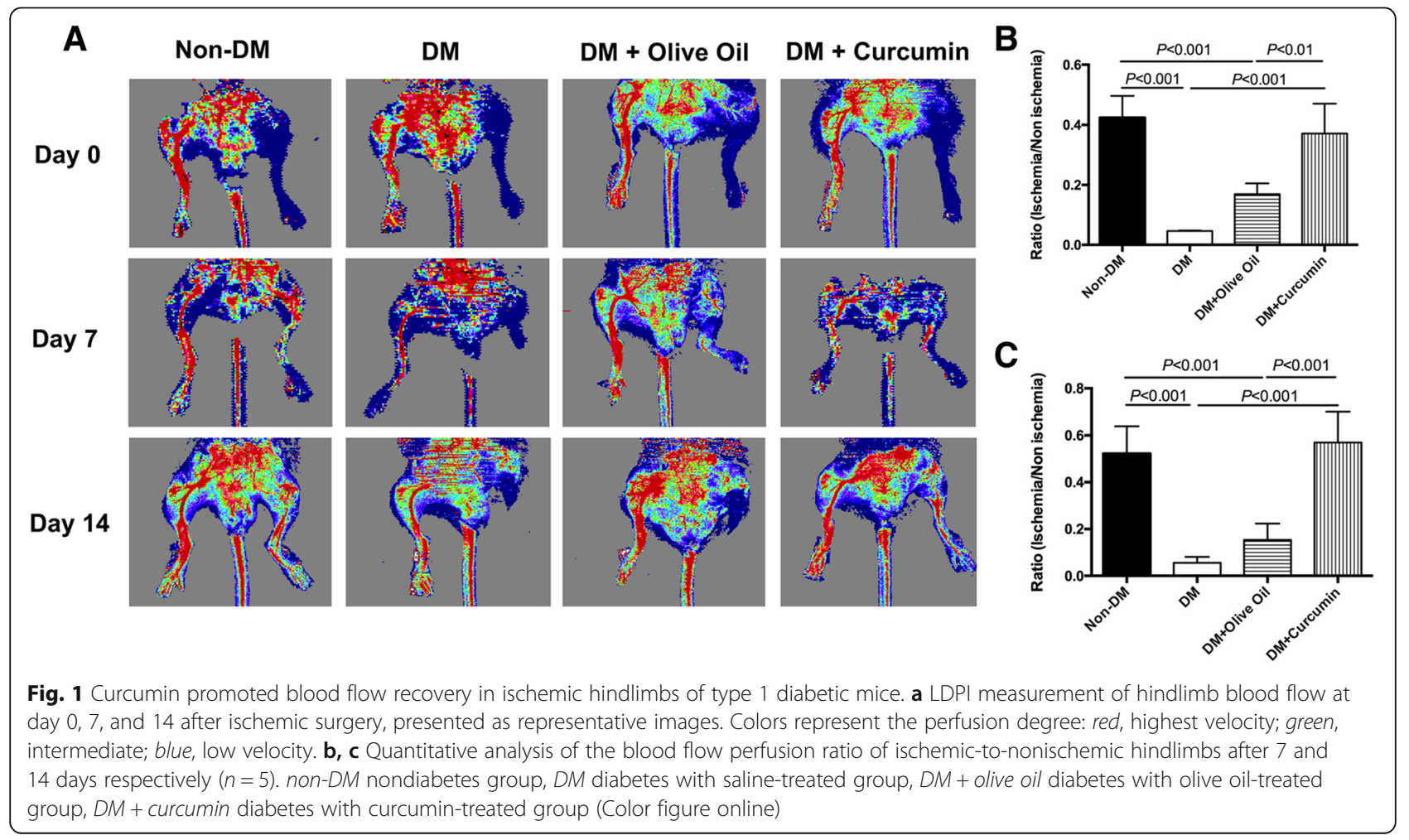



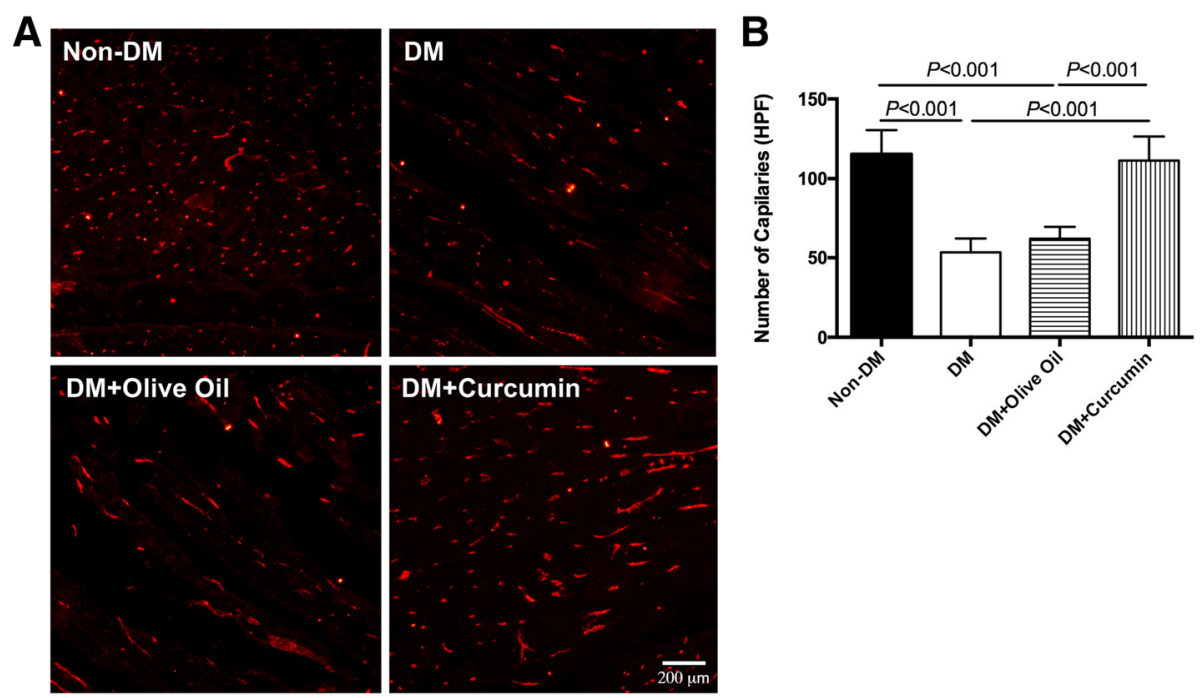

Fig. 2 Histological and quantitative analysis of ischemic hindlimb capillary density. a IB4-stained cells (red) were identified as neogenic capillaries. Bar, $200 \mu \mathrm{m}$. b Numbers of capillaries were counted and presented as mean \pm SD $(n=4)$. HPF high-power field, non-DM nondiabetes group, DM diabetes with saline-treated group, DM + olive oil diabetes with olive oil-treated group, DM + curcumin diabetes with curcumin-treated group (Color figure online)

shown in Fig. 4a, EPCs in untreated groups presented impaired tube incorporation capacity compared with that in non-EPCs isolated from diabetic mice. Precisely, $42.20 \pm 4.76 / \mathrm{HPF}$ incorporated EPCs were observed in the olive oil group, whereas $75.60 \pm 6.31$ /HPF incorporated EPCs were observed in the curcumin-treated group (Fig. 4b). This assay revealed that the curcumin-treated diabetic group showed better EPC incorporation into the new tubes than the olive oil-treated diabetic group.
Reversed senescence of EPCs isolated from diabetic mice by curcumin application

EPC senescence is increased under hyperglycemia condition [26]. To test whether curcumin could reverse the accelerated senescence of EPCs, we conducted a SA- $\beta$ gal assay. The senescent cells could be visualized in blue (Fig. 5a), and the senescence ratio of EPCs is presented in Fig. $5 \mathrm{~b}(P<0.05$; olive oil group, $43.32 \pm 1.83 \%$ vs curcumin treatment, $20.33 \pm 5.90 \%)$. These data indicate
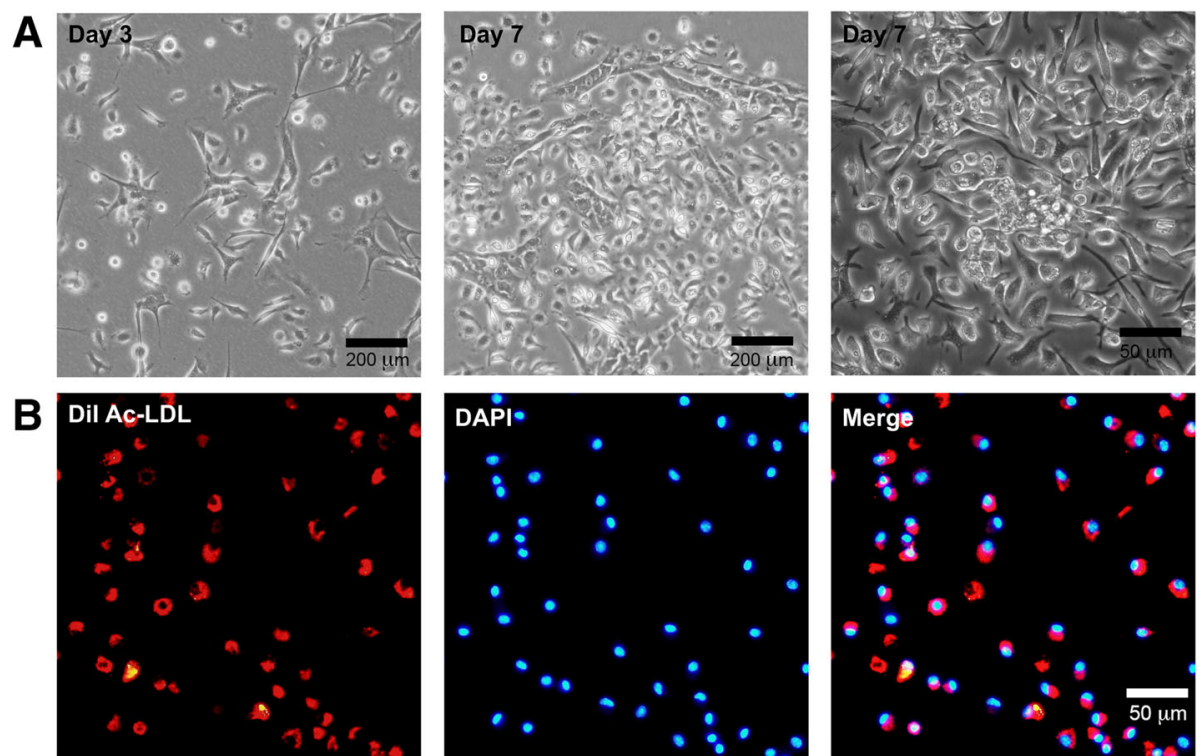

Fig. 3 Characterization of BM-derived EPCs. a Spindle-shaped EPCs could be observed 3 days after plating BM-MNCs. EPCs showed cobblestone-like morphology at day 7. Bar, $200 \mu \mathrm{m}$ and $50 \mu \mathrm{m}$. b EPCs uptaking Dil-Ac-LDL could be visualized (red). Nucleus (blue) was stained by DAPI. Bar, $50 \mu \mathrm{m}$ (Color figure online) 

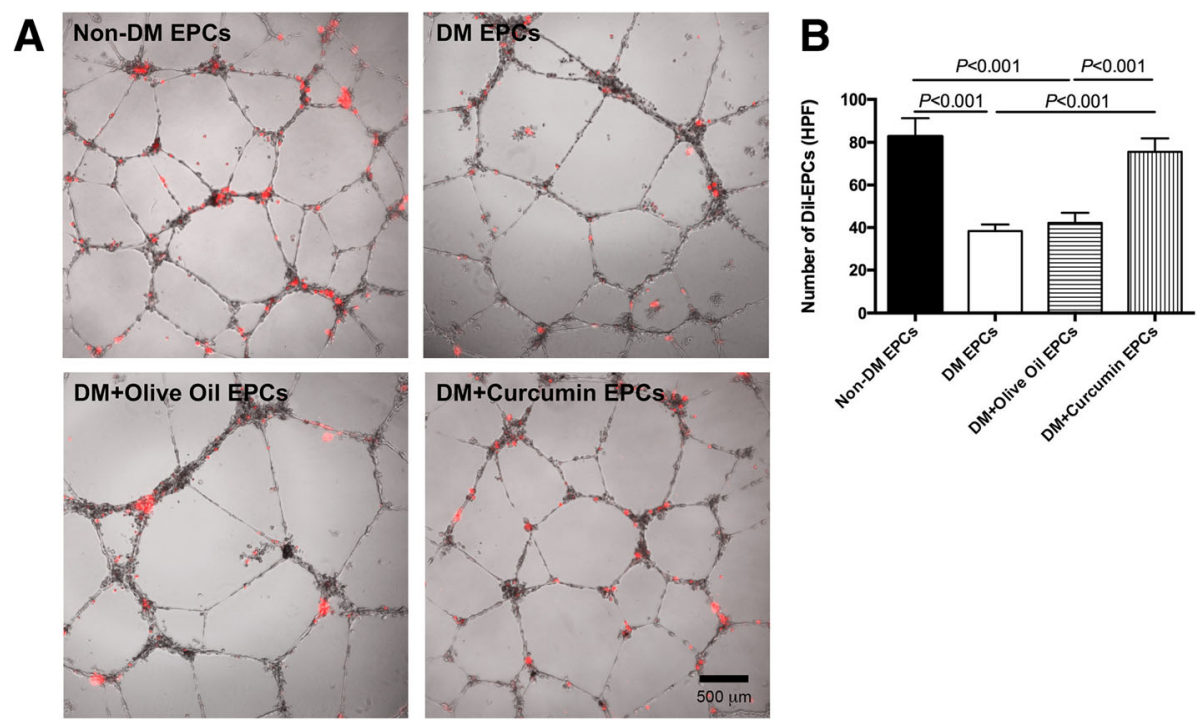

Fig. 4 Tube incorporation ability of EPCS isolated from diabetic mice was rescued by curcumin. a Morphology images of tube-formed HUVECs incorporated with Dil-positive EPCs isolated from diabetic mice (red). Bar, $500 \mu \mathrm{m}$. b Number of Dil-positive incorporated EPCs was counted and presented as mean \pm SD $(n=4)$. EPC endothelial progenitor cell, HPF high-power field, non-DM nondiabetes group, DM diabetes with saline-treated group, DM + olive oil diabetes with olive oil-treated group, DM + curcumin diabetes with curcumin-treated group (Color figure online)

that curcumin may have the potential to reverse the senescence of EPCs isolated from diabetic mice.

\section{Promoted migratory ability of curcumin-treated EPCs iso- lated from diabetic mice}

The transwell system was introduced to study the migration ability of EPCs isolated from diabetic mice. Cells were stained by DAPI and observed in HPFs (Fig. 6a). After treatment with curcumin, EPCs isolated from diabetic mice manifested an augment in migration compared with control groups $(P<0.05$; non-DM, $61.50 \pm 5.20 / \mathrm{HPF}$; DM, $18.50 \pm 2.65 / \mathrm{HPF} ; \mathrm{DM}+$ olive oil, $19.50 \pm 2.52 / \mathrm{HPF} ; \mathrm{DM}+$ curcumin, $63.00 \pm 5.29 / \mathrm{HPF}$ ). This assay indicated that curcumin had the potential to enhance the migration ability of EPCs isolated from diabetic mice.

\section{Enhanced proliferation ability of EPCs isolated from diabetic mice by curcumin}

The colony-formation ability of EPCs, representing the proliferation ability, was determined after curcumin
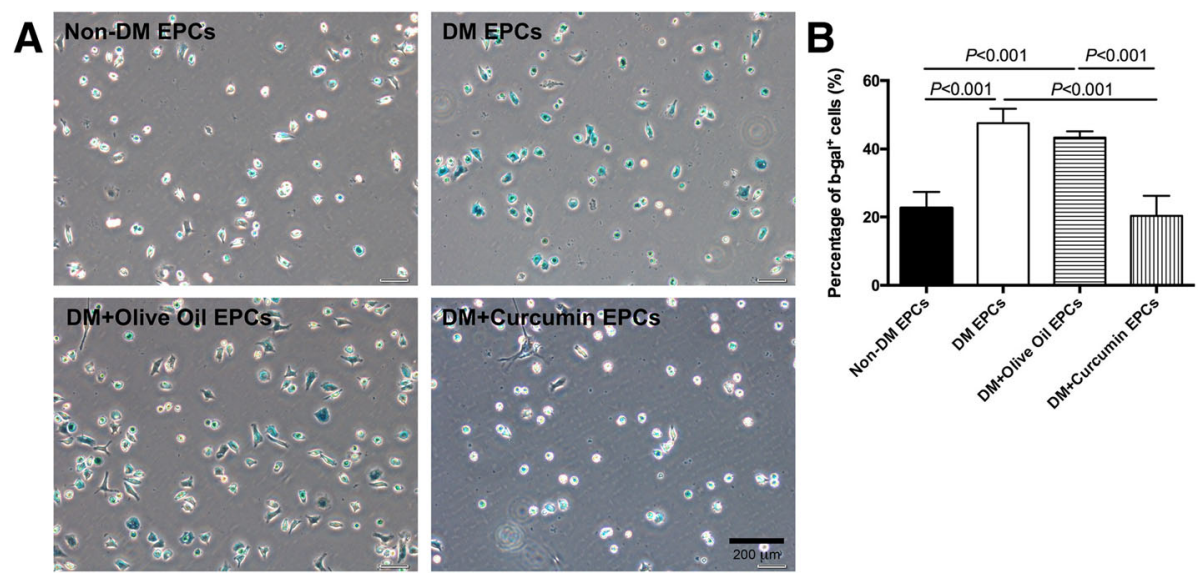

Fig. 5 Senescence of EPCs studied using $\beta$-galactosidase staining agent. a Representative images of SA- $\beta$-Gal-positive cells (blue). Bar, $200 \mu$ m. $\mathbf{b}$ Percentage of $\beta-G a^{+}$cells to total cells was calculated manually and presented as mean $\pm S D(n=4)$. EPC endothelial progenitor cell, non-DM nondiabetes group, DM diabetes with saline-treated group, DM + olive oil diabetes with olive oil-treated group, DM + curcumin diabetes with curcumintreated group (Color figure online) 
application. EPCs in the colonies could uptake DiI-AcLDL (Fig. 7a). Curcumin application increased the number of colonies by about twofold compared with untreated groups, without reaching a significant difference compared with the non-DM group (Fig. 7b; non-DM, $42.00 \pm 9.90 ; \mathrm{DM}, 16.50 \pm 3.87 ; \mathrm{DM}+$ olive oil, $16.50 \pm$ 3.87; DM + curcumin, $36.25 \pm 3.30 ; P<0.05$ ).

\section{qPCR validation of VEGF-A and Ang-1 expression}

VEGF-A and Ang-1 are considered the key proangiogenic factors [27]. Thus, expression of VEGF-A (Fig. 8a) and Ang-1 (Fig. 8b) was further analyzed by RT-qPCR. Curcumin could upregulate the expression of VEGF-A and Ang-1 in EPCs isolated from diabetic mice.

\section{Discussion}

In the present study, we demonstrated the curative effects of curcumin on ischemic hindlimbs in diabetic mice via promoting the function of EPCs isolated from diabetic mice. Assays and data exhibited that curcumin application to diabetic ischemia had the potential of: significantly promoting blood flow recovery via enhanced neovascularization; enhancing EPC function, namely angiogenesis, migration, and proliferation ability; reversing the hyperglycemia-induced EPC senescence; and upregulating the angiogenic factors, in terms of VEGF-A and Ang-1, in EPCs.

Kaushik et al. [28] reported spontaneous amputation of lower extremities in diabetes, demonstrating the high risk of necrosis and amputation in DM associated with PAD. In this study, more than half of the ischemic diabetic mice in untreated groups suffered from autoamputation by day 14, while the other half of these mice had different levels of necrosis (Additional file 1: Figure S1), which is in accordance with the above report. In contrast, curcumin application significantly rescued hindlimb ischemia in diabetic mice. The immunofluorescent result indicated that the capillary density was augmented substantially in the curcumintreated group compared with the untreated DM groups. This result manifested the strong therapeutic effects of curcumin on hindlimb ischemia in diabetes mellitus. We also observed that after the application of curcumin to euglycemic hindlimb ischemic mice, blood flow recovery and the neogenic vessels presented no significant difference from the control group (Additional file 2: Figure S2).

EPCs are involved in the neovascularization under hyperglycemic condition [29, 30]. Based on the in-vivo findings, we proposed that curcumin could take effect via modulating the functions of EPCs in diabetic ischemia. As a follow-up study, we isolated EPCs 14 days after curcumin application and directly detected their functions. We observed that curcumin reversed the impaired angiogenesis, migration, and proliferation abilities and the accelerated senescence of EPCs, and could hypothesize the hindlimb ischemia might be rescued by the augmented functions of EPCs. In order to exclude the potential effects of STZ on murine EPCs, we applied STZ directly to EPCs isolated from healthy mice. There were almost no promotional or inhibitory effects on the proliferation, tube formation, and migration abilities of the cells by STZ (Additional file 3: Figure S3).
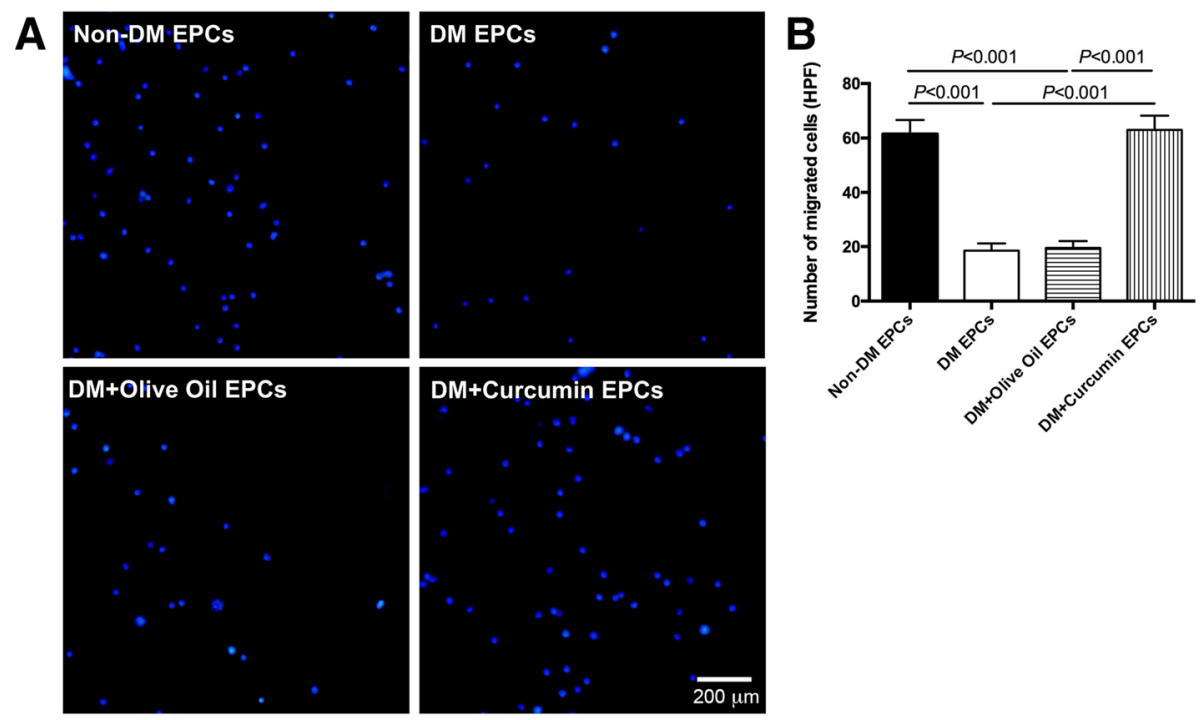

Fig. 6 Transwell assay to detect the migratory ability of EPCs isolated from diabetic mice. a Representative images show DAPI-stained migrated cells (blue) taken by fluorescent microscope. Bar, $200 \mu \mathrm{m}$. b Number of migrated cells was calculated manually and presented as mean \pm SD $(n=4)$. EPC endothelial progenitor cell, HPF high-power field, non-DM nondiabetes group, DM diabetes with saline-treated group, DM + olive oil diabetes with olive oil-treated group, DM + curcumin diabetes with curcumin-treated group (Color figure online) 

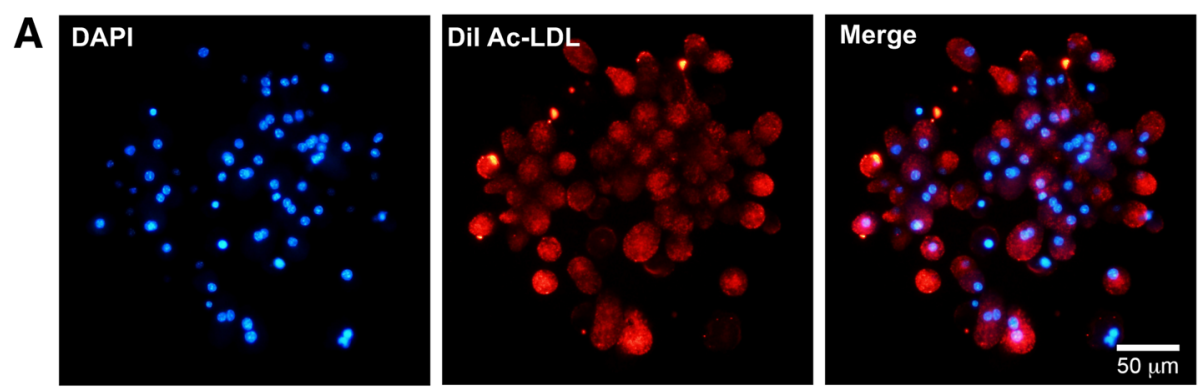

B

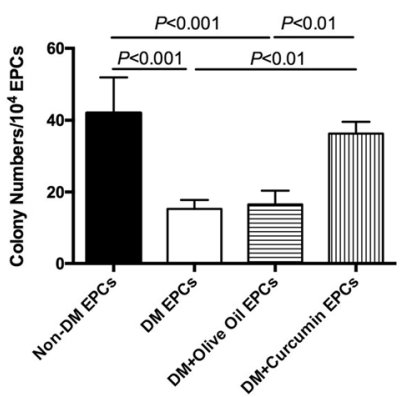

Fig. 7 Colony-formation assay showed curcumin could reverse the proliferation ability of EPCs isolated from diabetic mice. a Representative staining for Dil-Ac-LDL in EPC colonies. Bar, $50 \mu \mathrm{m}$. b EPC colonies were counted and analyzed in each group. Number of EPCs presented as mean \pm SD $(n=4)$. EPC endothelial progenitor cell, non-DM nondiabetes group, DM diabetes with saline-treated group, DM + olive oil diabetes with olive oil-treated group, DM + curcumin diabetes with curcumin-treated group (Color figure online)

One important finding in this study is that curcumin could upregulate the expression of VEGF-A and Ang-1 in EPCs. Kant et al. [11] observed curcumin-induced upregulation of VEGF expression in diabetic wounds. On the contrary, Shehzad et al. and Shan et al. [13, 14] observed that curcumin could downregulate VEGF expression in cancer cells. To explore the reason of these opposite results, Kiran et al. [11] further applied curcumin to endothelial cell culture in serum-free and serum-supplemented conditions, respectively. This study indicates that curcumin presents different effects depending on its microenvironment. Specifically, when cells are exposed to growth factors, curcumin may present an antiangiogenic effect; when cells are in a microenvironment that lacks exogenous stimuli, curcumin may have a proangiogenic effect [27]. Accordingly, we determined the mRNA level of VEGF and Ang-1 in hindlimb muscle collected from diabetic and nondiabetic mice. The mRNA expression level of both VEGF and Ang-1 was lower in the diabetic group than in the nondiabetic group (Additional file 4: Figure S4; VEGF, $722.9 \pm$
A

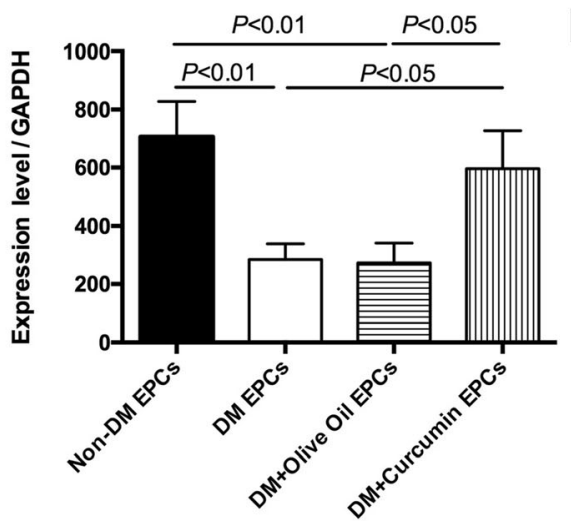

B

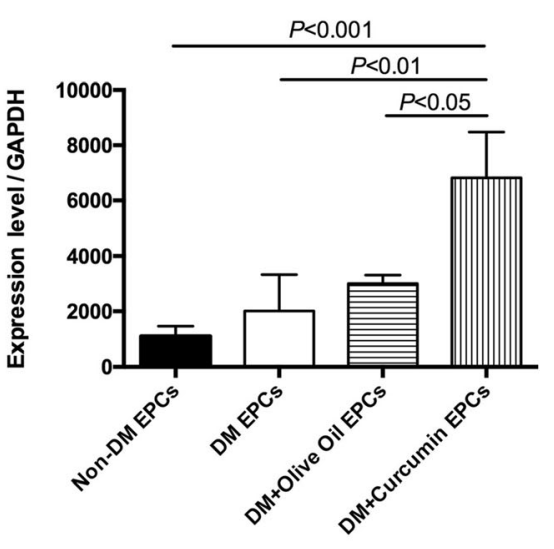

Fig. 8 qPCR analysis of neovascularization factors in different EPC groups. mRNA level of (a) VEGF-A and (b) Ang-1 determined by quantitative real-time RT-PCR. GAPDH used for the normalization of mRNA expression $(n=3)$. EPC endothelial progenitor cell, non-DM nondiabetes group, DM diabetes with saline-treated group, DM + olive oil diabetes with olive oil-treated group, DM + curcumin diabetes with curcumin-treated group 
140.6 in non-DM vs $390.7 \pm 121.3$ in DM; Ang- $1,365.8 \pm$ 135.6 in non-DM vs $106.8 \pm 13.9$ in DM; $P<0.05$ ), indicating a lack of proangiogenic factors in the diabetic microenvironment. To sum up, the microenvironment in hyperglycemia lacks exogenous stimuli factors [2, 3], which explains the phenomenon that in our study curcumin upregulated VEGF expression in EPCs, presenting a proangiogenic effect.

\section{Conclusion}

The results of this study reveal that lavage application of curcumin could ameliorate the decreased blood flow reperfusion in diabetic mice. In addition, EPCs isolated from curcumin-treated diabetic mice showed enhanced ability in migration, angiogenesis, and proliferation, and decreased senescence. Furthermore, VEGF-A and Ang-1 expression was significantly upregulated, which may be induced by curcumin treatment. In conclusion, curcumin manifested its therapeutic effects on the diabetic ischemic mice model, and could possibly be envisioned as a promising therapeutic method in treating diabetic ischemic diseases in human beings.

\section{Additional files}

Additional file 1: Figure S1. is showing gross appearance of mouse hindlimb ischemia model on day 14. Mice were anesthetized and photographs were taken at day 14. (JPG $3289 \mathrm{~kb}$ )

Additional file 2: Figure S2. is showing blood flow and the capillary density in ischemic hindlimbs of euglycemic mice treated with or without curcumin. (A) LDPI measured hindlimb blood flow at day 7 and 14 after ischemic surgery, presented as representative images. Colors represent the perfusion degree: red, highest velocity; green, intermediate; blue, low velocity. (B, C) Quantitative analysis of blood flow perfusion ratio of ischemic-tononischemic hindlimb after 7 and 14 days respectively $(n=3)$. LDPI results presented no difference between the group treated with curcumin and the control group. (D) IB4-stained cells (red) were identified as neogenic capillaries. Bar, $200 \mu \mathrm{m}$. (E) Numbers of capillaries were counted and presented as mean $\pm \mathrm{SD}(n=3)$. non-DM, non-diabetes group; non-DM + curcumin, nondiabetes group treated with curcumin. (JPG $2372 \mathrm{~kb}$ )

Additional file 3: Figure S3. is showing tube incorporation ability (A), migratory ability (B), and colony formation ability (C) of EPCs isolated from euglycemic mice treated with or without STZ. (A) Number of Dil-positive incorporated EPCS was counted and presented as mean \pm SD $(n=4)$. Tube incorporation ability of EPCS treated with STZ presented no difference with the control group. (B) Number of migrated cells was calculated manually and presented as mean \pm SD $(n=4)$. EPCs treated with STZ presented similar migratory ability to the control group. (C) EPC colonies counted and analyzed in each group. Number of EPCs colonies presented as mean \pm SD $(n=4)$. STZ streptozotocin. (JPG $1294 \mathrm{~kb}$ )

Additional file 4: Figure S4. is showing qPCR analysis of VEGF and Ang-1 in diabetic and nondiabetic mice hindlimbs. Total mRNA level of (A) VEGF-A and (B) Ang-1 determined by quantitative real-time RT-PCR. GAPDH was used for the normalization of mRNA expression $(n=3)$. (JPG $1841 \mathrm{~kb})$

\section{Abbreviations}

Ang-1: Angioprotein-1; BM: Bone marrow; BM-MNC: Bone marrow mononuclear cell; DM: Diabetes mellitus; EPC: Endothelial progenitor cell; HPF: High-power field; LDPI: Laser Doppler perfusion imaging; PAD: Peripheral arterial disease; SD: Standard deviation; VEGF: Vascular endothelial growth factor

\section{Acknowledgements}

Not applicable.

\section{Funding}

This work was supported by the National Natural Science Foundation of China (No. 81400199 and No. 81600217), Natural Science Foundation of Jiangsu Province (BK20160346), and Suzhou Municipal Science and Technology Project of China (No. SYS201414).

\section{Availability of data and materials}

The datasets supporting the conclusions of this article are included within the article.

\section{Authors' contributions}

$J Z Y, J S, T M, Z Y, X W, Z Z, J L$, and LW were responsible for performance of the experiment, data analysis, and manuscript preparation. ZS, JJY, and MI were responsible for manuscript writing and revision, and experimental design. All authors read and approved the final manuscript.

\section{Authors' information}

$J Z Y, J S, T M, Z Y, X W, Z Z, J L, L W, J J Y$, and ZS are with the Institute for Cardiovascular Science \& Department of Cardiovascular Surgery of The First Affiliated Hospital, Soochow University, Suzhou, China. MI is with the Division of Research Animal Laboratory and Translational Medicine, Osaka Medical College, Osaka, Japan.

\section{Ethics approval}

The experiment protocols were approved by the Ethic Committee of Soochow University (reference number: SZUM2008031233).

\section{Consent for publication}

All authors have contributed to, read, and approved the final manuscript for submission.

\section{Competing interests}

The authors declare that they have no competing interests.

\section{Publisher's Note}

Springer Nature remains neutral with regard to jurisdictional claims in published maps and institutional affiliations.

\section{Author details}

${ }^{1}$ Department of Cardiovascular Surgery of the First Affiliated Hospital \& Institute for Cardiovascular Science, Soochow University, Suzhou, China. 2Division of Research Animal Laboratory and Translational Medicine, Osaka Medical College, Osaka, Japan.

Received: 14 March 2017 Revised: 22 June 2017

Accepted: 21 July 2017 Published online: 03 August 2017

\section{References}

1. Jude EB, et al. Peripheral arterial disease in diabetic and nondiabetic patients: a comparison of severity and outcome. Diabetes Care. 2001;24(8):1433-7.

2. Bitto $A$, et al. Angiopoietin-1 gene transfer improves impaired wound healing in genetically diabetic mice without increasing VEGF expression Clin Sci (Lond). 2008;114(12):707-18.

3. Kampfer $\mathrm{H}$, Pfeilschifter J, Frank S. Expressional regulation of angiopoietin-1 and -2 and the tie- 1 and -2 receptor tyrosine kinases during cutaneous wound healing: a comparative study of normal and impaired repair. Lab Invest. 2001;81(3):361-73.

4. Altabas V. Diabetes, Endothelial dysfunction, and vascular repair: what should a diabetologist keep his eye on? Int J Endocrinol. 2015;2015:848272.

5. Asahara T, et al. Isolation of putative progenitor endothelial cells for angiogenesis. Science. 1997;275(5302):964-7.

6. Ceradini DJ, et al. Decreasing intracellular superoxide corrects defective ischemia-induced new vessel formation in diabetic mice. J Biol Chem. 2008; 283(16)::10930-8

7. Isner JM, Asahara T. Angiogenesis and vasculogenesis as therapeutic strategies for postnatal neovascularization. J Clin Invest. 1999;103(9):1231-6. 
8. Fadini GP, et al. Circulating endothelial progenitor cells are reduced in peripheral vascular complications of type 2 diabetes mellitus. J Am Coll Cardiol. 2005;45(9):1449-57.

9. Yiu KH, Tse HF. Specific role of impaired glucose metabolism and diabetes mellitus in endothelial progenitor cell characteristics and function. Arterioscler Thromb Vasc Biol. 2014;34(6):1136-43.

10. Li Y, et al. In mice with type 2 diabetes, a vascular endothelial growth factor (VEGF)-activating transcription factor modulates VEGF signaling and induces therapeutic angiogenesis after hindlimb ischemia. Diabetes. 2007;56(3):656-65.

11. Kant $\mathrm{V}$, et al. Curcumin-induced angiogenesis hastens wound healing in diabetic rats. J Surg Res. 2015;193(2):978-88.

12. Sun $L N$, et al. Curcumin attenuates high glucose-induced podocyte apoptosis by regulating functional connections between caveolin-1 phosphorylation and ROS. Acta Pharmacol Sin. 2016;37(5):645-55.

13. Shehzad A, Lee J, Lee YS. Curcumin in various cancers. Biofactors. 2013:39(1):56-68.

14. Shan $B$, et al. Curcumin suppresses HIF1A synthesis and VEGFA release in pituitary adenomas. J Endocrinol. 2012;214(3):389-98.

15. Kalka C, et al. Transplantation of ex vivo expanded endothelial progenitor cells for therapeutic neovascularization. Proc Natl Acad Sci U S A. 2000;97(7):3422-7.

16. Mimeault M, Batra SK. Frequent deregulations in the hedgehog signaling network and cross-talks with the epidermal growth factor receptor pathway involved in cancer progression and targeted therapies. Pharmacol Rev. 2010;62(3):497-524.

17. Zhao T, Li J, Chen AF. MicroRNA-34a induces endothelial progenitor cell senescence and impedes its angiogenesis via suppressing silent information regulator 1. Am J Physiol Endocrinol Metab. 2010;299(1):E110-6.

18. Hamada $\mathrm{H}$, et al. Estrogen receptors alpha and beta mediate contribution of bone marrow-derived endothelial progenitor cells to functional recovery after myocardial infarction. Circulation. 2006;114(21):2261-70.

19. Yang J, et al. CD34+ cells represent highly functional endothelial progenitor cells in murine bone marrow. PLoS One. 2011;6(5):e20219.

20. Qin Y, et al. Sonic hedgehog improves ischemia-induced neovascularization by enhancing endothelial progenitor cell function in type 1 diabetes. Mol Cell Endocrinol. 2016;423:30-9.

21. Zhong $\mathrm{W}$, et al. L-arginine attenuates high glucose-accelerated senescence in human umbilical vein endothelial cells. Diabetes Res Clin Pract. 2010;89(1):38-45.

22. Livak KJ, Schmittgen TD. Analysis of relative gene expression data using real-time quantitative PCR and the 2(-Delta Delta C(T)) method. Methods. 2001;25(4):402-8.

23. Yang J, et al. Regulatory roles of interferon-inducible protein 204 on differentiation and vasculogenic activity of endothelial progenitor cells. Stem Cell Res Ther. 2016;7(1):111.

24. Sekiguchi $\mathrm{H}$, et al. Improved culture-based isolation of differentiating endothelial progenitor cells from mouse bone marrow mononuclear cells. PLoS One. 2011;6(12):e28639.

25. Asai J, et al. Topical sonic hedgehog gene therapy accelerates wound healing in diabetes by enhancing endothelial progenitor cell-mediated microvascular remodeling. Circulation. 2006;113(20):2413-24.

26. Yuan $\mathrm{Q}$, et al. Accelerated onset of senescence of endothelial progenitor cells in patients with type 2 diabetes mellitus: role of dimethylarginine dimethylaminohydrolase 2 and asymmetric dimethylarginine. Biochem Biophys Res Commun. 2015;458(4):869-76.

27. Kiran MS, et al. Opposing effects of curcuminoids on serum stimulated and unstimulated angiogenic response. J Cell Physiol. 2008;215(1):251-64.

28. Kaushik R, Sree BS, Attri AK. Spontaneous auto-amputation of the foot in a case of diabetes, atherosclerosis and gangrene. J Indian Med Assoc. 2002; 100(9):573-4.

29. Costa PZ, Soares R. Neovascularization in diabetes and its complications. Unraveling the angiogenic paradox. Life Sci. 2013;92(22):1037-45.

30. Ebrahimian TG, et al. NADPH oxidase-derived overproduction of reactive oxygen species impairs postischemic neovascularization in mice with type 1 diabetes. Am J Pathol. 2006;169(2):719-28.

\section{Submit your next manuscript to BioMed Central and we will help you at every step:}

- We accept pre-submission inquiries

- Our selector tool helps you to find the most relevant journal

- We provide round the clock customer support

- Convenient online submission

- Thorough peer review

- Inclusion in PubMed and all major indexing services

- Maximum visibility for your research

Submit your manuscript at www.biomedcentral.com/submit
Biomed Central 